\title{
MALE BREAST CANCER
}

https://doi.org/10.29289/259453942021V31S1077

\section{MALE BILATERAL BREAST CANCER: CASE REPORT}

Maria Eduarda Bernardino Martins Melo1', Gabriela Prado Lopes, Darley Lima Ferreira Filho², Irnanda Layanna Gomes Oliveira ${ }^{1}$, Maria Eduarda Vasconcelos Florêncio Cavalcanti'

${ }^{1}$ Centro universitário Mauricio de Nassau - Recife (PE), Brazil.

${ }^{2}$ Hospital Barão de Lucena - Recife (PE), Brazil.

Introduction: Breast cancer occurring bilaterally in men is extremely rare. Breast cancer represents $1 \%$ of all cancers, while bilateral cancer represents $5 \%$ of a total number of patients with breast cancer, which may be synchronic or metachronic. Many cases of breast cancer in men are detected between 60 and 70 years, with an average of 67 years of age. In men there is a tendency for late diagnosis at a more advanced stage than in women. Case report: A male patient, JSS, 68 years old, from Afogados da Ingazeira, state of Pernambuco, was seen with breast tumoration in June 2016. He arrived at the service with an existing diagnosis of breast cancer through core-biopsy examination. The physical exam presented bulging in the left retroareolar region and a hardened tumoration in palpation. Radical mastectomy was performed. The histopathological results confirmed an invasive mucinous carcinoma with histological grade I, nuclear grade II and mitotic grade I. Free margins. The most frequent histological type in men is ductal (85\%-90\%), followed by papillary in $4.5 \%$ and mucinous in $2.8 \%$ of the cases. Nineteen free axillary lymph nodes were dissected, with Estrogen and Progesterone + receptors, Her-2, negative and with Ki-67 of 5\%. Breast cancers in men present with more positivity for hormone receptors and low expression for Her-2. The pathological staging was classified as II a. The patient was being followed by the clinical oncology department, where he was chosen not to do chemotherapy and only hormone therapy, with Tamoxifen $20 \mathrm{mg}$. However, over a period of six months he noticed the presence of a tumoration in the right breast. An image examination was performed with MG/USG, which confirmed the presence of a tumor in the right retro-areolar region (Birads IV). A core-biopsy of the lesion was requested, which confirmed an invasive breast cancer. The patient underwent right radical mastectomy, whose result confirmed an invasive ductal carcinoma with pathological staging II a. Conclusion: This pathology is extremely rare in men and the evaluation of the contralateral breast is of fundamental importance. Early diagnosis and compliance with treatment will reduce tumor recurrence and provide a better prognosis for these patients. 